\title{
Some Characteristics of Nocturnal Penile Tumescence during Puberty
}

\author{
Ismet Karacan ${ }^{[25]}$, Carolyn J. Hursah, Robert L. Williams, and Ramon C. Littell \\ Department of Psychiatry, University of Florida College of Medicine; Psychiatry Service, Veterans Administration Hospital; \\ and Department of Statistics, University of Florida, Gainesville, Florida, USA
}

Extract

This paper presents a study of nocturnal penile tumescence (NPT) and the relation of this phenomenon to stage 1 rapid eye movement (REM) sleep for a group of pubertal males. Ten subjects, ages 12.8 to 15.3 years, slept in the laboratory for three consecutive nights. Electroencephalograms, electrooculograms, and measurements of penile erections were recorded.

The average tumescence time per night was 158.99 min; average REM time was $137.07 \mathrm{~min}$. Average simultaneous REM and tumescence per night was $101.89 \mathrm{~min}$. These subjects averaged 6.85 tumescence episodes/night, and, of these, 5.15 occurred during a REM period. Tumescence episodes during REM averaged $30.77 \mathrm{~min}$ in duration, whereas episodes which occurred when no REM was present averaged 11.75 min. All subjects had at least four REM periods per night and at least three tumescence episodes. No subject had more than 6 REM periods or more than 11 tumescence episodes.

The amount of penile tumescence during a night's sleep was found to be less subject to the first night effect than was total sleep time, which indicates the strength of the underlying process controlling tumescence. Two components of NPT were described: a high probability component (maximum tumescence during REM), and a variable probability component (all nonmaximum and non-REM tumescence). Although the results indicated that there is a close relation between REM and tumescence, no simple cause and effect relation between the two phenomena was apparent.

NPT seems to be a complex process with multiple functions. Although it may play a role in sexual development, it may be only partially related to the sexual activity of the mature male.

\section{Speculation}

Nocturnal penile tumescence in pubertal males is temporally related to the appearance of REM activity during sleep. This tumescence represents a complex process with multiple functions, one of which may be sexual development.

\section{Introduction}

The regular occurrence of NPT was first noted by Ohlmeyer et al. [17, 18]. The suggestion by Aserinsky [2] that REM sleep and NPT might be temporally related, and preliminary work by Oswald [19] in showing that relation, encouraged others to launch detailed studies of these phenomena. Subsequently, Karacan [11] and Fisher et al. [5] succeeded in showing a high 
temporal relation between REM and NPT in young adults. Their work has since been confirmed by Jovanović [6].

Although some data have been reported regarding NPT characteristics in other age groups, e.g., among infants [12, 16] and among the elderly [10], a complete ontogenetic description of NPT has not yet been reported.

Our laboratory is studying NPT as a function of age. Our ultimate goal is to describe the ontogeny of NPT and to relate the variables of nocturnal tumescence to those of sleep stages, aging, and sexual development. In addition, base line data produced by this study may aid in the diagnosis and prognosis of clinical conditions such as retarded sexual development and sexual precocity.

Because of the profound neurophysiologic, hormonal, and psychologic changes associated with puberty, a description of NPT characteristics during this period is of special interest. Therefore, this study provides data on the characteristics of NPT in healthy males aged $12.8-15.3$ years.

\section{Methods and Procedures}

The subjects were 10 white male high school students in good health, ranging in age from 12.8 to 15.3 years (mean 14.6). A physical examination I year prior to the recording of sleep characteristics had revealed that none of the subjects had completed pubertal growth, as defined by Donovan and van der Werff ten Bosch [3]. Pubertal growth is characterized primarily by the development of primary and secondary sex characteristics, such as enlargement of gonads and development of body hair. At the time of the sleep and tumescence recordings, all subjects had pubertal growth, according to these criteria.

An electroencephalogram (EEG) and an electrooculogram (EOG) were recorded for each of the 10 subjects on 3 consecutive nights. Each subject reported to the laboratory $1.5 \mathrm{hr}$ prior to his accustomed bedtime. Each had been requested to abstain from coffee and other beverages containing caffeine, and to refrain from taking naps and excessive exercise after lunch that day.

Electrode placement, recording technique, and scoring procedures were those of Williams et al. [23], including as the criterion for $\delta$ wave amplitucle: peak to peak, $40 \mu \mathrm{v}$.

Penile erections were monitored with a mercuryfilled silicone elastic ring fitted over the penis behind the glans. During erections, stretching of the tubing and the corresponding resistance by the mercury cause a direct change in the calibrated amplitude on the EEG graph, so that duration and degree of each erectile episode are graphically recorded. Details concerning transducer reliability and penile tumescence measurement by our technique have been described [13, 15].

A deviation from the base line of the tumescence tracing is considered a tumescence episode if it lasts at least $20 \mathrm{sec}$ with an amplitude at least $20 \%$ of the greatest amplitude recorded for that subject. The $20 \%$ minimum eliminates minor deviations which are apparently unrelated to tumescence episodes. It was decided to tailor the criteria to the individual subject after examination of 2000 records from 300 normal aclults revealed that penile circumferences during maximum erection differ by as much as $3 \mathrm{~cm}$. Obviously, predetermined criteria for maximum circumferences would not be appropriate. Therefore, the criterion is based on the percentage variation from the greatest circumference recorded for each subject.

A deviation in amplitude on the tumescence tracing constitutes either a maximum or a semi-episode. For a maximum episode, circumference of the penis must be between 81 and $100 \%$ of the greatest circumference recorded for the subject, and, for a semi-episode, the circumference must be between 20 and $80 \%$ of this value. These criteria correspond with reports by subjects that they are unaware of deviations of $20 \%$ or less, that they are aware of some degree of tumescence during deviations of $20-80 \%$, and that they experience a full erection cluring deviations of $80-100 \%$.

Each maximum erectile episode consists of a point at which the circumference of the penis begins to increase (designated $T-u p$ ), a period of maximum circumference (T-max), a period of decrease (T-down), and, finally, the point at which the penis again becomes flaccid (T-zero). In semi-erections, these phases are scored in a similar manner, but, because the period of fullest tumescence is not comparable to that of a maximum erection, the phases of semi-erections are treated separately.

During an episode the penis may attain maximum tumescence for a while, become flaccid, then reattain some degree of tumescence. Such events are classified according to the length and degree of the transient period of flaccidity and the degree of tumescence reattained after this period. If the penis becomes completely flaccid for $1 \mathrm{~min}$ or longer, the event is classified as two distinct episocles. A period of complete 
flaccidity shorter than 1 min, or a period of unlimited length during which penis circumference is between 1 and $80 \%$ of the maximum, is called a fuctuation if the penis reattains $80 \%$ or more of its maximum circumference after the period of transient flaccidity.

The penis throbs, particularly during the $T$ - up phase. This appears on the recording as an irregular 1or 2-mm change in amplitude, which usually lasts 1 or $2 \mathrm{sec}$, and is called a pulsation.

Morning awakening often occurs during tumescence, resulting in an interrupted episode. These were not included in the analysis if distortion would result. For example, amount of tumescence and number of tumescence episodes per night were calculated with interrupted episodes included, but average lengths of episodes were calculated without inclusion of interrupted episodes. Similar procedures were used for REM periods terminated by morning awakening.

\section{Results}

The first night of EEG sleep recording is generally not included in data analysis because it has been shown [ 1 , 20] that first night data are significantly different from data obtained on succeeding nights. It is assumed that this reflects the subject's initial reaction to the laboratory situation. Therefore, with the exception of the comparisons reported immediately below, only data from nights 2 and 3 are reported here.

Two records, one for each of two subjects, were unscoreable. Therefore, data are reported for a total of 18 nights. The means for each variable were calculated over one or two nights of recording for each subject, and subject means were averaged to derive group means.

\section{First Night Effect}

Average sleep time for the first night of recording was $433.57 \mathrm{~min}$ and the average REM time was 102.20 min. Comparison (by $t$ tests for matched pairs unless stated otherwise) of these data with those of later nights (Table I) revealed that both total sleep time and total REM time were significantly shorter on the first night $(P<0.05)$ than on succeeding nights.

On the other hand, average tumescence time on the first night was not significantly different from the averages for subsequent nights, even though the range of subject means for total tumescence time on the first night (53.40-272.30 min) was greater than that on succeeding nights (111.40-267.80 min).
Table I. Average nightly tumescence time, REM time, and total sleep time for 10 subjects, $12.8-15.3$ years old ${ }^{1}$

\begin{tabular}{lrc}
\hline \multicolumn{1}{c}{ Variable } & $\begin{array}{c}\text { Mean/night, } \\
\text { min }\end{array}$ & $\begin{array}{c}\text { Range of } \\
\text { subject means }\end{array}$ \\
\hline Sleep time & 491.85 & $474.00-514.00$ \\
REM time & 137.07 & $114.25-162.45$ \\
Tumescence time & 158.99 & $111.40-267.80$ \\
Ratio, tumescence time/REM & 1.19 & $0.84-2.17$ \\
$\quad$ time & 0.32 & $0.22-0.52$ \\
$\quad \begin{array}{l}\text { Ratio, tumescence time/sleep } \\
\quad \text { time }\end{array}$ & 0.28 & $0.24-0.33$ \\
Ratio, REM time/sleep time & & \\
\end{tabular}

${ }^{1}$ REM : rapid eye movement.

\section{Relation between Major Variables}

Average sleep time, REM time, and tumescence time per night, and their relations are presented in Table I. Whereas the ranges of subject means for total sleep time (474.00-514.00 $\mathrm{min})$ and for REM time (114.25-162.45 $\mathrm{min}$ ) were fairly narrow, the range for tumescence time was wide: $111.40-267.80 \mathrm{~min}$. These ranges indicate individual differences among subjects.

Thirty-two per cent of total sleep time was spent in tumescence (range, 22-52\%); REM time averaged $28 \%$ of total sleep time (range, 24-33\%); and the ratio of tumescence time to REM time was 1.19 (range, $0.84-2.17)$.

\section{Length and Frequency of Tumescence Episodes}

In Table II are tabulated the average frequency and length of maximum episodes, of semi-episodes, and of all tumescence episodes (maximum and semi) together for: (1) episodes which occurred at least in part during REM, (2) episodes which occurred entirely during non-REM (NREM) sleep, and (3) all episodes, regardless of the sleep stage in which they occurred.

Subjects averaged 6.85 episodes/night, and these episodes averaged $27.04 \mathrm{~min}$ in length. Most episodes occurred at least partly during REM; REM episodes occurred an average of 5.15 times/night and lasted 30.77 min, whereas NREM episodes occurred only 1.70 times/night and lasted $11.75 \mathrm{~min}$. All 10 subjects had at least 3 REM episodes, and 8 subjects had at least 1 NREM episode.

Maximum episodes during REM, with a frequency of 4.90 times/night and an average length of 31.22 min, were characteristic of this age group. All $10 \mathrm{sub}$ jects had them.

Semi-tumescence episodes during REM were observed in only two subjects. The group averaged 0.25 
Table II. Average number and length of maximum and semi-tumescence episodes during REM and NREM for 10 subjects, 12.815.3 years old ${ }^{1}$

\begin{tabular}{|c|c|c|c|c|c|c|}
\hline & \multicolumn{2}{|c|}{ Maximum episodes } & \multicolumn{2}{|c|}{ Semi-episodes } & \multicolumn{2}{|c|}{ All episodes } \\
\hline & No. ${ }^{2}$ & Length, min & No. ${ }^{2}$ & Length, min & $N_{0.2}^{2}$ & Length, min \\
\hline During REM & $4.90(1.29)$ & $31.22^{2} \quad(12.25)$ & $0.25(0.63)$ & $3.60^{3} \quad(1.70)$ & $5.15(1.53)$ & $30.77^{2}(12.78)$ \\
\hline During NREM & $1.40(1.33)$ & $12.62^{4} \quad(5.52)$ & $0.30 \quad(0.42)$ & $7.54^{5}(5.58)$ & $1.70(1.30)$ & $11.75^{6} \quad(5.44)$ \\
\hline During all sleep stages & $6.30(2.37)$ & $28.25^{2}(11.46)$ & $0.55(0.96)$ & $5.97^{5}(2.72)$ & $6.85(2.57)$ & $27.04^{2}(12.10)$ \\
\hline
\end{tabular}

Values a re expressed as means; the standard deviation appears in parentheses. REM : rapid eye movement; NREM: non-REM. 1 Mean of 10 subjects.

2 Mean of 2 subjects.

3 Mean of 7 subjects.

4 Mean of 4 subjects.

5 Mean of 8 subjects.

6

Table III. Temporal relations between REM and tumescence for 10 subjects, $12.8-15.3$ years old ${ }^{1}$

\begin{tabular}{|c|c|c|c|}
\hline Event & No. & $\%$ & Amount/night, $\min ^{2}$ \\
\hline All tumescence episodes & 119 & & \\
\hline Tumescence episodes accompanied by REM & 90 & 75.6 & \\
\hline All REM periods & 87 & & \\
\hline REM periods accompanied by tumescence & 79 & 90.8 & \\
\hline \multicolumn{4}{|l|}{ Initial onset times ${ }^{3}$} \\
\hline REM onset preceded $T-u p$ & 47 & 52.2 & \\
\hline$T$-up preceded REM onset & 38 & 42.2 & \\
\hline REM onset coincided with $T-u p$ & 5 & 5.6 & \\
\hline Total & 90 & 100.0 & \\
\hline \multicolumn{4}{|l|}{ Phase onset times ${ }^{4}$} \\
\hline REM onset preceded $T$-max & 65 & 76.5 & \\
\hline$T$-max preceded REM onset & 19 & 22.3 & \\
\hline REM onset coincided with $T-\max$ & 1 & 1.2 & \\
\hline Total & 85 & 100.0 & \\
\hline REM onset preceded $T$-down & 73 & 92.4 & \\
\hline$T$-down preceded REM onset & 6 & 7.6 & \\
\hline REM onset coincided with $T$-down & 0 & 0 & \\
\hline Total & 79 & 100.0 & \\
\hline \multicolumn{4}{|l|}{ Stop times ${ }^{3}$} \\
\hline REM stop preceded $T$-zero & 47 & 52.2 & \\
\hline T-zero preceded REM stop & 33 & 36.7 & \\
\hline REM stop coincided with $T$-zero & 2 & 2.2 & \\
\hline Interrupted episodes ${ }^{5}$ & 8 & 8.9 & \\
\hline Total & 90 & 100.0 & \\
\hline \multicolumn{4}{|l|}{ Simultaneous REM-tumescence } \\
\hline Maximum episodes & & & $100.81(23.51)$ \\
\hline Semi-episodes & & & $1.08 \quad(3.02)$ \\
\hline All episodes & & & $101.89(22.16)$ \\
\hline
\end{tabular}

${ }^{1}$ REM : rapid eye movement; $T$-up: period of increasing penile circumference; $T$-max: period of maximum penile circumference;

$T$-down: period of decreasing penile circumference; $T$-zero: flaccid penis.

${ }^{2}$ Means; standard deviation appears in parentheses.

${ }^{3}$ For all REM-related episodes.

${ }^{4}$ For REM maximum episodes only.

${ }^{5}$ Episodes which terminated with subject's awakening in the morning.

such episodes/night and, when they occurred, they lasted an average of only $3.60 \mathrm{~min}$.

NREM maximum episodes were observed in 7 of the 10 subjects. They had an average occurrence of 1.40 times/night for the group. NREM semi-episodes were observed in only four of the subjects, with an average occurrence, for the group, of 0.30 times/night. NREM maximum episodes had a mean length of $12.62 \mathrm{~min}$, whereas this value was $7.54 \mathrm{~min}$ for NREM semi-episodes. 


\section{Temporal Relation between REM and Tumescence}

There were 119 tumescence episodes during the 18 nights of the study (Table III). Of these, 90 , or $75.6 \%$, occurred at least partly during a REM period. The other $24.4 \%$ occurred entirely during NREM. There were 87 REM periods in all, and, of these, 79, or $90.8 \%$, were accompanied by at least one phase of a tumescence episode. The remaining eight REM periods were not accompanied by tumescence.

For $52.2 \%$ of the 90 NPT episodes accompanied by REM, onset of REM preceded T-up; in $42.2 \%$, onset of $T$-up preceded onset of REM; and for $5.6 \%$ of the episodes, onset of REM and tumescence coincided.

When the onsets of $T$-max and $T$-down phases are compared with initial REM onset, it is found that REM onset preceded $T$-max onset and $T$-down onset in 76.5 and $92.4 \%$ of the episodes, respectively.

The end of the REM period (REM stop) preceded the end of the accompanying tumescence episode ( $T$ zero) in most cases $(52.2 \%)$.

\section{Simultaneous REM-Tumescence}

Simultaneous REM-tumescence is that time during which REM and tumescence are both occurring. Subjects spent an average of $101.89 \mathrm{~min} /$ night in this condition. When simultaneous REM-tumescence time is compared with tumescence time (Table I), it is apparent that $64 \%$ of tumescence was simultaneous REMtumescence.

Most simultaneous REM-tumescence was maximum tumescence. Subjects spent $100.81 \mathrm{~min} /$ night (or 63\% of total tumescence time) in this condition. Simultaneous REM-semi tumescence occupied $1.08 \mathrm{~min} /$ night (or $1 \%$ of total tumescence time).

\section{Lengths of Phases}

In Table IV are presented the average lengths of the phases of maximum tumescence episodes and these lengths as percentages of the total average phase length. T-up phase during REM was about one-sixth as long as $T$-max, and $T$-max was almost twice as long

Table $I V$. Average length of the three phases of maximum tumescence episodes for 10 subjects, $12.8-15.3$ years old ${ }^{1}$

\begin{tabular}{|c|c|c|c|c|c|c|}
\hline \multirow{2}{*}{ Type of episode } & \multicolumn{3}{|c|}{$\begin{array}{l}\text { Leng th of phase, } \\
\text { min }\end{array}$} & \multicolumn{3}{|c|}{$\begin{array}{c}\text { Length of phase as } \\
\% \text { of average episode length }\end{array}$} \\
\hline & $T-u p$ & $T-\max$ & $T$-down & $T-u p$ & $T-\max$ & $T$-down \\
\hline During REM ${ }^{2}$ & $3.18(1.58)$ & $18.19(9.05)$ & $9.70(4.12)$ & 10.2 & 58.5 & 31.2 \\
\hline During NREM ${ }^{3}$ & $1.78(1.12)$ & $4.55(4.37)$ & $6.29(2.80)$ & 14.1 & 36.1 & 49.8 \\
\hline During all sleep stages ${ }^{2}$ & $2.86(1.10)$ & $16.16(8.51)$ & $9.26(3.81)$ & 10.1 & 57.1 & 32.7 \\
\hline
\end{tabular}

'Values are expressed as means; standard deviation appears in parentheses. REM : rapid eye movement; NREM : non-REM; T-up: period of increasing penile circumference; $T$-max: period of maximum penile circumference; $T$-down: period of decreasing penile circumference.

2 Means of values for 10 subjects.

${ }^{3}$ Means of values for 7 subjects.

Table $V$. Average lengths of successive REM periods and tumescence episodes, and number of fluctuations and pulsations for successive tumescence episodes for 10 subjects, 12.8-15.3 years old ${ }^{1}$

\begin{tabular}{|c|c|c|c|c|c|c|c|c|c|c|}
\hline \multirow{3}{*}{$\begin{array}{l}\text { RFM period } \\
\text { or tumescence } \\
\text { episode number }\end{array}$} & \multirow{3}{*}{$\begin{array}{l}\text { No. of } \\
\text { subjects }\end{array}$} & \multirow{2}{*}{\multicolumn{2}{|c|}{$\begin{array}{l}\text { REM periods } \\
\text { Length, min }\end{array}$}} & \multirow{3}{*}{$\begin{array}{l}\text { No. of } \\
\text { subjects }\end{array}$} & \multicolumn{6}{|c|}{ Tumescence episodes } \\
\hline & & & & & \multicolumn{2}{|c|}{ Length, min } & \multicolumn{2}{|c|}{ No. of fluctuations } & \multicolumn{2}{|c|}{ No. of pulsations } \\
\hline & & Mean & $\mathrm{SD}^{2}$ & & Mean & SD & Mean & SD & Mean & SD \\
\hline 1 & 10 & 19.84 & 9.81 & 10 & 26.34 & 14.66 & 0.40 & 0.66 & 0.60 & 0.74 \\
\hline 2 & 10 & 33.08 & 13.97 & 10 & 33.82 & 16.99 & 0.75 & 0.98 & 3.10 & 3.24 \\
\hline 3 & 10 & 33.91 & 16.76 & 10 & 30.55 & 12.74 & 0.40 & 0.66 & 5.45 & 7.29 \\
\hline 4 & 10 & 33.97 & 10.20 & 9 & 22.76 & 12.22 & 1.22 & 1.44 & 2.94 & 4.19 \\
\hline 5 & 5 & 23.55 & 10.42 & 8 & 22.59 & 17.28 & 1.37 & 1.83 & 2.00 & 4.47 \\
\hline 6 & 1 & 9.70 & & 7 & 16.56 & 18.93 & 0.43 & 0.79 & 1.00 & 1.38 \\
\hline 7 & & & & 5 & 9.45 & 3.83 & 0.40 & 0.89 & 0.10 & 0.22 \\
\hline 8 & & & & 3 & 13.10 & 8.06 & 1.00 & 1.00 & 0.00 & \\
\hline 9 & & & & 2 & 16.85 & 14.78 & 2.50 & 3.54 & 0.00 & \\
\hline 10 & & & & 2 & 9.15 & 4.45 & 0.50 & 0.71 & 0.00 & \\
\hline 11 & & & & 2 & 8.85 & 8.70 & 0.00 & & 0.00 & \\
\hline
\end{tabular}

1 REM : rapid eye movement.

${ }^{2} \mathrm{SD}$ : standard deviation. 
Table VI. Tumescence episodes and REM periods for various age groups ${ }^{2}$

\begin{tabular}{|c|c|c|c|c|c|c|c|c|c|c|c|c|}
\hline & \multicolumn{12}{|c|}{ Age, yr } \\
\hline & \multicolumn{2}{|c|}{$13-15$} & \multicolumn{2}{|c|}{$20-26$} & \multicolumn{2}{|c|}{$30-37$} & \multicolumn{2}{|c|}{$41-46$} & \multicolumn{2}{|c|}{$51-59$} & \multicolumn{2}{|c|}{$60-69$} \\
\hline & $\begin{array}{c}\text { Fre- } \\
\text { quency }\end{array}$ & $\underset{\min }{\text { Amount }}$ & $\begin{array}{c}\text { Fre- } \\
\text { quency }\end{array}$ & $\underset{\min }{\text { Amount, }}$ & $\begin{array}{c}\text { Fre- } \\
\text { quency }\end{array}$ & $\underset{\min }{\text { Amount, }}$ & $\begin{array}{c}\text { Fre- } \\
\text { quency }\end{array}$ & $\underset{\text { min }}{\text { Amount, }}$ & $\begin{array}{c}\text { Fre- } \\
\text { quency }\end{array}$ & $\underset{\min }{\text { Amount }}$ & $\begin{array}{c}\text { Fre- } \\
\text { quency }\end{array}$ & $\underset{\text { min }}{\text { Amount, }}$ \\
\hline $\begin{array}{l}\text { Tumescence epi- } \\
\text { sodes }\end{array}$ & 6.85 & 158.99 & 4.22 & 131.45 & 4.53 & 136.89 & 3.80 & 110.84 & 3.90 & 112.32 & 3.53 & 88.45 \\
\hline REM periods & 4.85 & 137.07 & 4.06 & 113.09 & 4.83 & 102.22 & 4.30 & 90.12 & 4.75 & 101.42 & 5.02 & 93.48 \\
\hline $\begin{array}{l}\text { Simultaneous } \\
\text { REM-tumes- } \\
\text { cence }\end{array}$ & & 101.89 & & 87.22 & & 73.99 & & 52.61 & & 56.76 & & 50.97 \\
\hline
\end{tabular}

1 Values are expressed as nightly averages. REM : rapid eye movement.

as $T$-down. Also, during NREM $T$ - $u p$ was shorter than $T$-max, but $T$-down exceeded the length of $T$-max.

During REM, $T-u p$ occupied only $10.2 \%$ of the episode length. $T$-max predominated, occupying $58.5 \%$; and $T$-down constituted $31.2 \%$ of the episode length. During NREM, the $T$-up phase was also the smallest portion $(14.1 \%)$ of the total, whereas $T$-max occupied $36.1 \%$, and $T$-down was approximately half $(49.8 \%)$ of the episode length.

\section{Cycles and Intervals}

A cycle is the average time from the beginning of one tumescence episode to the beginning of the next. An interval is the average time between two episodes. The average cycle lasted $72.23 \mathrm{~min}$, and the mean interval between episodes was $43.48 \mathrm{~min}$.

\section{Lengths of Successive REM Periods and Tumescence Episodes, and Number of Fluctuations and Pulsations}

All subjects averaged at least 4 REM periods per night (Table V). Lengths of REM periods increased progressively through the fourth period, whereas the tendency was to decrease thereafter. Only five subjects had a fifth period and only one subject had a sixth.

Two subjects had as many as 11 tumescence episodes a night and all subjects had at least 3 episodes/night. The mean length for the first episode was $26.34 \mathrm{~min}$. Length started to decrease with the third episode. The 11 th episode averaged $8.85 \mathrm{~min}$. No pattern is apparent in the number of fluctuations. However, the pattern for number of pulsations was similar to that for episode length. There was an increase in the number of pulsations from the first through the third episode, and then a decrease. There were no pulsations at all in the last four episodes. It should be noted that the standard deviations for all fluctuation and pulsation measures were high.

\section{Comparison with Other Age Groups}

In Table VI, frequency and duration of tumescence episodes, REM periods, and simultaneous REM-tumescence are compared for age groups from approximately 13 through 69 years. With increasing age there is a decline in the absolute amount of all three variables, and the greatest decrease is in tumescence. Only the number of REM periods per night remains constant, there is little change across the entire age range. In contrast, the number of tumescence episodes appears to peak in the 13- to 15-year-old group, with an abrupt decrease in 20- to 26-year-old group and little change after that.

\section{Discussion}

The fact that there was no significant difference between the amount of time spent in tumescence on the first night and on succeeding nights suggests that nocturnal penile tumescence during puberty is under the control of a process which is resistant to the psychologic factors thought to be present during the first night in the laboratory. However, the wide range of subject means indicates that there are marked individual differences in degree of development of the underlying process which controls nocturnal tumescence.

The rarity and brevity of semi-episodes in the subjects may indicate the stength of the underlying process. Most episodes reached maximum tumescence. The few which did not were brief. However, it seems that the coincidence of REM somelow prolonged the tumescence episode, inasmuch as the average maximum episode during REM was more than twice as long as the average maximum episode during NREM. In other words, the process which controls tumescence seems to be operating under reduced effectiveness during NREM sleep. Semi-tumescence episodes, on the other hand, showed the reverse tendency: they were longer 
during NREM than during REM. However, since only two subjects had semi-episodes during REM and only four subjects had them during NREM, these results may not be representative.

A review of the literature demonstrates that the amount of REM-related tumescence varies considerably with the age group of the subjects and with the investigator. For example, Korner [16] reported that, in a group of 32 full term white neonates, $39 \%$ of all REM periods were accompanied by erections. However, Karacan [12] reported that $84 \%$ of the REM periods of 15 Negro neonates were accompanied by erections. Karacan et al. [14] reported that $80 \%$ of REM periods of 16 subjects in their early 20's were accompanied by tumescence, and Fisher et al. [4, 5] observed that $95 \%$ of the REM periods of a group of 20 - and 30 -year-old subjects were accompanied by tumescence. Kahn and Fisher $[8,10]$ point out that there is a great deal of variability in the percentage of REM-related erections in older males, and that 5 of 12 subjects between the ages of 71 and 80 had amounts similar to that of a young adult. Jovanović [7] reported $70-98 \%$ REM-related erections in a group of 20 males 19-50 years of age. Recent data collected in our laboratory indicate that there is a consistent decrease in the amount of simultaneous REM-tumescence from age 13 years through age 69 years. For the group of pubertal males, $21 \%$ of sleep period time (time from onset of sleep until final awakening) was spent in simultaneous REM-tumescence; for ages 20-26, 30-37, $41-46,51-59$, and 60-69, this percentage was 20, 17, 13, 13 , and 12, respectively. An analysis of variance performed on these data indicated a significant difference with $P<0.05$.

The differences between these reports of REM-related tumescence may be due to different methods of observation or measurement, or both, but some are certainly due to age and methodologic differences. Some of the discrepancies may be due to a failure to discriminate among the four types of tumescence which we have described (REM-max, REM-semi, NREM-max, NREM-semi) since these types were not differentiated by other investigators.

The stability of the NPT phenomenon is roughly comparable to that of other measurable sleep variables, but, from the results presented here, it seems to be more variable than does REM for this age group.

However, the standard deviations of the NPT are nearly all small in relation to the mean. This indicates that, although there are individual differences in
NPT, the magnitude of these differences is not excessively large.

In Table V, a close similarity to REM is evident: all 10 subjects had at least 4. REM periods and all 10 subjects had at least 3 NPT episodes. Therefore, it is apparent that in this age group NPT occurs with about the same minimum frequency per night as REM. Whether this stability improves or decreases with age is one of the questions to be answered by the normative data which we are now collecting.

This study indicates that NPT includes two components: a high probability component and a variable probability component which are, respectively, maximum tumescence during REM and all nonmaximum and non-REM tumescence. Although all subjects exhibited REM maximum episodes on all nights of the study, not all subjects had the other three types of episodes, nor did a subject who had one such episode on one night necessarily exhibit that type on all other nights.

While this study indicates that there is a relation between REM and tumescence (specifically, $90.8 \%$ of REM periods were accompanied by erections), certainly there was no apparent cause and effect relation between the two phenomena in this group of pubertal males. The beginning of tumescence preceded the begining of REM almost as often as the reverse (Table III). It appears that both were responding to the same underlying process, and it was almost pure chance which activity, REM or tumescence, started first.

These findings are in line with our position that REM and tumescence are both surface indicators of the periodic bursts of autonomic activity to which mentation, in the form of dreams, has also been closely linked. There is evidence that the parameters of these indicators undergo some changes with age. For example, Roffwarg et al. [21] reported a decrease in the amount of REM sleep from $25 \%$ during young adulthood to $13-18 \%$ in old age. However, Webb and Agnew [22] were unable to find a significant decrease in REM sleep with age, and Kahn and Fisher [9] found $21.7 \%$ REM in 71 - to 90 -year-old subjects and lower percentages after age 90 . Recent data from our laboratory show that the amounts of time spent in tumescence and in REM are both susceptible to change with increasing age. For the group of pubertal males, $32 \%$ of sleep period time was spent in tumescence. For ages 20-26, 30-37, 41-46, 51-59, and 60-69, this percentage was $30,31,28,24$, and 20 , respectively; REM time constituted $28,26,23,22,23$, and $22 \%$ of 
sleep time, respectively. Analysis of variance performed on each of these variables indicated significant differences with $P<0.05$.

If the periodic burst of autonomic activity which is reflected in REM and NPT serves some vital maintenance function which the organism requires to a different degree at different age levels, then it may be, as our data suggest, that tumescence is a more sensitive indicator of this need than is REM.

If, in addition, NPT has some specific sexual function, then this would explain why there was more tumescence than REM in these subjects, and why the NREM maximum episode was a fairly frequent occurrence. In other words, it is possible that, until puberty, NPT is either responding to this new function or is contributing to it, or both.

Examination of the tumescing $(T-u p)$, tumescent (T-max), and detumescing ( $T$-down) phases of a REM maximum episode reveals that, for pubertal males, the majority of tumescence time is spent in the $T$-max phase with the penis remaining in full erection (Table IV). Recent findings in our laboratory suggest that this is not true for older males. Adult subjects spent the majority of time in the detumescing phase.

When the lengths of successive episodes in Table $\mathrm{V}$ are examined, it is seen that for this group of young males there is no consistent increase or decrease across episodes. However, our adult age groups had a more stable pattern of increasing length up to the third or fourth episode, after which there was a fairly consistent decrease through the last episode. This suggests that the subjects in this study are undergoing a process of maturation and psychosexual development, that there are considerable individual differences in the degree of this development, and that the variability of successive episode lengths reflects these individual differences.

Our current view is that NPT is a complex process with multiple functions. In childhood it may serve to stimulate and therefore develop the psychosexual cortex, whereas in adult life it may serve only as a maintaining function for the already developed cortex. Although it may play a role in sexual development, it may be only partially related to the sexual activity of the mature male. Its persistent connection with REM periods indicates that it is part of a general excitatory process which occurs at regular intervals during sleep. Although the predictability of the occurrence of a maximum tumescence episode during REM is high, it is as yet unknown whether the entire process of NPT is an expression of sexual excitation, or whether noc- turnal tumescence is an incidental result of this ongoing process. Our dream research, coupled with tumescence data and finer REM period analyses, are expected to shed light on this question. In addition, when norms are established for infants, children, and adults, this phenomenon may be useful as an indicator of the level of sexual development in the male. If NPT can be shown to be such an indicator, the established norms may be used to develop objective methods for diagnosing and evaluating psychosexual disorders.

\section{Summary}

The nocturnal penile tumescence (NPT) of a group of pubertal males was examined in detail and compared with that in groups of older males ranging in age from 20 to 69 years. The temporal relation of NPT and REM periods was also examined. The functional significance of NPT and its correspondence with REM were considered.

\section{References and Notes}

1. Agnew, H. W., Webb, W. B., and Williams, R. L.: The first night effect: an EEG study of sleep. Psychophysiology, 2: 263 (1966).

2. Aserinsky, E.: Ocular motility during sleep and its application to the study of rest-activity cycles and dreaming. Unpublished doctoral dissertation, University of Chicago, 1953.

3. Donovan, B. T., and van der Werff ten Bosch, J. J.: Physiology of Puberty. (Williams \& Wilkins, Baltimore, 1965).

4. Frsher, C.: Dreaming and sexuality. In: R. M. Loewenstein, L. M. Newman, M. Schur, and A. J. Solnit: PsychoanalysisA General Psychology. (International Universities Press, New York, 1966).

5. Fisher, C., Gross, J., ANd Zuch, J.: Cycle of penile erection synchronous with dreaming (REM) sleep. Arch. Gen. Psychiat., 12: 29 (1965).

6. Jovanović, U.: Erectionen im Schlaf. Arch. Psychiat. Nervenkr., 210: 220 (1967).

7. Jovanović, U. J.: Die Periodik der Erektionen im Schlaf. Med. Klin., 63: 923 (1968).

8. KAHN, E., AND Fisher, C.: REM sleep and sexuality in the aged. J. Geriat. Psychiat., 2: 181 (1969).

9. KaHn, E., AND Fisher, C.: The sleep characteristics of the normal aged male. J. Nerv. Ment. Dis., 148 : 477 (1969).

10. KaHN, E., AND Fisher, C.: Some correlates of rapid eye movement sleep in the normal aged male. J. Nerv. Ment. Dis., 148 . 495 (1969).

I1. Karacan, I.: The effect of exciting presleep events on dream reporting and penile erection during sleep. Unpublished doctoral dissertation, New York University, 1965.

12. KARACAN, I.: The developmental aspect and the effect of certain clinical conditions upon penile erection during sleep. Excerpta Med. Int. Congr. Ser., 150: 2356 (1966).

13. KaraCAN, I.: A simple and inexpensive transducer for quantitative measurements of penile erection during sleep. Behav. Res. Meth. Instrum., 1: 251 (1969). 
14. Karacan, I., Goodenough, D., and Shapiro, A.: Some psychological and physiological correlates of penile erection during sleep. Paper presented at the Fifth Annual Meeting of the Association for the Psychophysiological Study of Sleep, Washington, D.C., 1965.

15. Karacan, X., Hursch, C. J., AND Wrlliams, R. L.: Some characteristics of nocturnal penile tumescence in young adults. Arch. Gen. Psychiat., 26: 351 (1972).

16. Korner, A.: REM organization in neonates. Arch. Gen. Psychiat., 19: 330 (1968).

17. Ohilmeyer, P., and Brilmayer, H.: Periodische Vorgänge im Schlaf: II Mitteilung. Pfluegers Arch. Gesamte Physiol. Menschen Tiere, 249: 50 (1947).

18. Ohlmeyer, P., Brilmayer, H., and Hullstrung, H.: Periodische Vorgänge im Schlaf. Pfluegers Arch. Gesamte Physiol. Menschen Tiere, 485: 559 (1944).

19. Oswal.d, I.: Sleeping and Waking. (Elsevier, New York, 1962).

20. Rechtschaffen, A., AND Verdone, P.: Amount of dreaming: effect of incentive, adaptation to laboratory, and individual differences. Percept. Mot. Skills, 19: 947 (1964).
21. Roffwarg, H., Dement, W., and Fisher, C.: Preliminary observations of the sleep-dream pattern in neonates, infants, children, and adults. In: E. Harms: Problems of Sleep and Dream in Children. (Pergamon, New York, 1964).

22. Webs, W. B., ANd Agnew, H. W.: Measurement and characteristics of noctural slcep. In: L. Abt and B. Reiss: Progress in Clinical Psychology, Vol. 8. (Grune and Stratton, New York, 1968).

23. Williams, R. L., Agnew, H. W., And Webb, W. B.: Sleep patterns in young adults: an EEG study. Electroencephalogr. Clin. Neurophysiol., 17: 376 (1964).

24. Supported by Public Health Service Research Grant no. MH 15508, Veterans Administration Research Grant Funds, Clinical Research Center FR-82, and Fellowship to Senior Investigator.

25. Requests for reprints should be addressed to: IsMET KARACAN, M.D., Department of Psychiatry, University of Florida, Gainesville, Fla. 32601 (USA).

26. Accepted for publication Deccmber 17, 1971. 